\title{
Energy Efficiency and Influencing Factor Analysis in the Overall \\ Chinese Textile Industry
}

\author{
Lihong Peng ${ }^{\text {a* }}$, Yiting Zhang ${ }^{a}$, Yejun Wang ${ }^{\mathrm{b}}$, Xiaoling Zeng ${ }^{\mathrm{a}}$, Najun Peng ${ }^{\mathrm{a}}$, and Ang Yu ${ }^{\mathrm{a}}$ \\ ${ }^{\mathrm{a}}$ College of the Environment \& Ecology, Xiamen University, Xiang'an South Road, Xiang'an \\ District, Xiamen, 361102, China
}

${ }^{\mathrm{b}}$ School of Chinese Language and Literature, Jimei University, Yinjiang Road, Jimei District, Xiamen, 361021, China

\begin{abstract}
This study analyzes the overall textile industry (OTI) energy efficiency gap between China and the US and measures the longitudinal changes in OTI energy efficiency over time. A breadthwise comparison of the energy efficiency among OTI subsectors indicates that the chemical fiber industry (CFI) is the key subsector for improving energy efficiency. This study conducts a quantitative analysis of the influencing factors of energy efficiency of the CFI in China. The energy efficiency of the CFI in Fujian and its influencing factors are compared with that of the national level. Results indicate that the main factors influencing the energy efficiency of CFI in China include economic structure, energy structure, industry scale, and technology, whereas industry concentration has no significant effect. Economic indicators show that the energy efficiency of
\end{abstract}

\footnotetext{
*Corresponding author: Lihong Peng. Address: Room A119, College of the Environment \& Ecology, Xiamen University, Xiang'an South Road, Xiang'an District, Xiamen, 361102, P. R. China. Tel: (86)-139-50123497; Fax: (86)-592-2181916.

E-mail addresses: lhpeng@xmu.edu.cn (L.H. Peng); tinazytxmu@163.com (Y.T. Zhang); wangyj@jmu.edu.cn (Y.J. Wang); xlzeng@stu.xmu.edu.cn (X.L. Zeng); keepeng@xmu.edu.cn (N.J. Peng); yuang@xmu.edu.cn (A. Yu)

Abbreviations: overall textile industry (OTI); chemical fiber industry (CFI); textile industry (TI); garments, shoes, and hats manufacturing (GSHM); industrial added value (IAV); energy used per unit of production value (PV); added value (AV); energy consumption per unit product (EP); energy consumption per unit of industrial added value (EAV); energy consumption per unit of production value (EPV); economic system variable (ESV); energy structure (ES); industry concentration ratio (ICR); industry scale of a sector (IS); technical level (TL).
} 
Fujian's CFI has continued to improve yearly, and is currently above the national average. The low proportion of state-owned and state holding enterprises value, low proportion of coal consumption in the energy structure, industrial scale with a majority of medium- and large-sized enterprises, and advanced technology level are the main reasons for the success of Fujian's CFI. Finally, this study proposes several feasible policies that could be adopted by the authorities concerned.

Keywords: Overall textile industry; Chemical fiber industry; Energy efficiency; Influencing Factor; China; Fujian

\section{Introduction}

Data from the International Energy Agency (IEA) show that approximately 28\% of energy consumption in 2011 can be attributed to industrial production on a global scale (IEA, 2012), whereas this figure reached as high as 70\% in China (International Energy Workshop or IEW, 2013). The Intergovernmental Panel on Climate Change (IPCC, 2007) reported that carbon dioxide $\left(\mathrm{CO}_{2}\right)$ emissions caused by global energy consumption increased from $20 \mathrm{GtCO}_{2}$ in 1990 to $26 \mathrm{GtCO}_{2}$ in 2005. In addition, $36 \%$ of $\mathrm{CO}_{2}$ emission was released from energy-intensive industries, such as steel production, paper making, and textile manufacturing (IEA, 2007). These industrial sectors dominate China's total $\mathrm{CO}_{2}$ emissions at $72 \%$ in 2010 (IEW, 2013).

China is the largest textile manufacturer in the world, generating close to two-thirds of all textile fibers produced worldwide in 2012, followed by India and the United States (Lenzing, 2013). The overall textile industry (OTI) is an energy-intensive and high-emission industry with an energy consumption and greenhouse gas emission that ranks relatively high among the manufacturing sectors. In 2010, the final energy consumption of OTI, including three subsectors, namely, textile industry (TI), chemical fiber industry (CFI), and garments, shoes, and hats manufacturing industry (GSHM) accounted for $4.10 \%$ of the total energy consumption in China (DES, 2011).

Fujian is one of the major OTI provinces in China and is representative of the TI, CFI, and 
GSHM. In 2010, OTI industrial output accounted for $12 \%$ of that of the province, with the OTI economic aggregate of Fujian ranking fifth in the country. The comprehensive energy consumption of OTI in Fujian was also significant, ranking seventh among all energy-intensive industries in the province (FPBS, 2012).

The Chinese government, in its “Twelfth Five-year (2011-2015) Plan for National Economic and Social Development Outline", clearly indicated that the energy consumption per unit of GDP should be reduced by $16 \%$ by 2015 with the aim of achieving energy conservation and emissions reduction. Fujian Province also promulgated the “Twelfth Five-year Development Plan for Fujian's Textile Industry", which provides a guide for the sustainable development of the local OTI. This plan suggests that the industrial added value (IAV) of textile enterprises above the designated size should grow at an average annual rate of $16 \%$, whereas the energy consumption per unit of IAV should decline by $20 \%$ of the 2010 levels. These aims undoubtedly pose considerable challenges to the OTI of China.

Energy use and carbon emissions are closely linked. $\mathrm{CO}_{2}$ emissions from fossil-based energy consumption account for $89 \%$ of the total $\mathrm{CO}_{2}$ emissions in China (Zhao et al, 2013). Studies have shown that improving energy efficiency is the most direct means of reducing greenhouse gas emissions (Mohsen and Akash, 1998). Energy efficiency serves an important function in reducing fossil energy consumption, which in turn can reduce air pollution and deter human-induced climate change (Blesl et al., 2007). An industry is the basic starting point of national energy strategies. Studies on industrial energy efficiency and its main driving factors from the perspective of global and local industries will provide essential information for the establishment of reasonable energy policies. As an energy-intensive industry, OTI is an important contributor to $\mathrm{CO}_{2}$ emissions. Hence, investigating the energy efficiency of OTI is necessary to reduce the energy consumption of the industry and achieve energy conservation and emissions reduction targets.

This study aims to contribute to the understanding of energy use and energy efficiency in 
Chinese OTI through multi-level comparisons and analyses. The gap between China and foreign countries, such as the US, will be examined to assess the energy efficiency performance of OTI from both the industry and subsector levels in China, conduct a longitudinal analysis of the status quo of energy efficiency of Chinese OTI, and perform a transverse comparison of the energy efficiencies of the different subsectors. Energy efficiency will be represented using the appropriate metrics. This paper also focuses on the subsector with the highest unit energy consumption and on key areas such as the Fujian Province. This study conducts a quantitative analysis of the influencing factors of the energy efficiency of the key subsector in China and the differences in the factors in Fujian Province. The results of multi-level comparisons and analysis of the main influencing factors of energy efficiency in the key subsector of China and Fujian will enable the proposal of several feasible policies. In investigating the energy consumption and utilization problem of the Chinese OTI from the national, sub-industrial, and provincial levels, this study has the following aims: to provide guidance for the sustainable energy utilization of the OTI, to serve as the basis for further energy conservation, and to provide a scientific reference for decisions related to energy management.

\section{Methods}

\subsection{Literature overview}

International research on energy efficiency started early and hence is more systematic and substantial than that in China. Trianni et al. (2013) investigated several barriers that hinder the adoption of energy-efficient technologies and practices in foundries in European countries. Cui et al. (2014) calculated the energy efficiencies of nine countries using the economic value-added method. Utlu and Hepbasli (2007) analyzed the energy efficiencies of countries by reviewing studies conducted on various countries or societies. Tanaka (2008) assessed various energy efficiency performance measures in the industry and the application of these measures in policies. Sue Wing and Eckaus (2008) found that the aggregate energy-GDP ratio of an industry decomposes into shifts 
in sectoral/subsectoral composition (structural/product structural change) and adjusts according to the industry energy demand (efficiency change). Their results showed that in the prophase, adjusting the industrial structure improved energy efficiency, but the internal efficiency of an industry had clearer effects on energy efficiency in the long run. In China, most existing studies on energy efficiency focused on the national or regional level (Wang et al, 2013; Wu et al., 2014; Zhang and Lan, 2013), and only few on industrial energy efficiency. Therefore, most research objects in literature are mostly on national or regional energy efficiency, whereas industrial energy efficiency as a research object is relatively rare.

Although OTI is a vital industry sector with significant energy consumption, few scientific papers have addressed energy efficiency issues in this industry. Palanichamy and Babu (2005) acknowledged the fruitful energy conservation experiences of the Indian TI and proposed the potential of increased energy efficiency. Ozturk (2005) estimated the energy consumption per production for the textile sector and surveyed the relationship among energy usage, energy cost, and textile production. Martínez (2009) adopted the energy used per unit of production value (PV), which signifies the market value of all the final goods and services produced by a country or region in a given period, and added value (AV), which refers to the new value added during the production of industrial enterprises, to describe the development of energy efficiency in German and Colombian industrial sectors. Palamutcu (2010) calculated the actual and estimated energy consumption using production ratios of electric energy the various stages of the cotton textile process, such as spinning, warping sizing, weaving, wet processing, and clothing manufacturing in Turkey. Hong et al. (2010) used the energy per unit of the gross output value and the GDP to show the energy utilization status of 303 firms in the Taiwanese TI. Reddy and Ray (2011) examined the ratio of energy consumption to production as the efficiency indicator in five industrial sectors (iron and steel, aluminum, textiles, paper, and cement) in India and found that the combined effect of structure and efficiency was positive for the aluminum and textile sectors. 
Cagno and Trianni (2012) also identified the most effective energy efficiency practices in the manufacture of primary metals, plastics, and textiles in both small- and medium-sized enterprises in North America and Italy. Hasanbeigi (2010) provided more than 190 technologies and measures for energy efficiency, which were applicable to TI. Hasanbeigi and Price (2012a) proposed further improvement opportunities for energy efficiency, which are available in some major textile subsectors and proposed that the most aggregated energy efficiency was at the industry level, followed by the industry subsector level, which can reflect the general industry-wide or subsector-wide efficiency level. Hasanbeigi et al. (2012b) selected the indicator energy use/unit of product as the metric and compared 13 textile plants from five major subsectors of the Iranian TI to show that energy saving/management efforts should be focused on motor-driven systems. Hasanbeigi et al. (2012b) also emphasized that future research in textile must focus on the conduct of energy efficiency studies in countries where TI energy use is significantly high, such as in China.

However, existing research in China is limited. Liao and Wei (2010) indicated that without significant changes in the energy input structure, economic indicators could be used simply and feasibly to measure energy efficiency. Chen (2013) selected three subsectors, namely, TI, CFI, and GSHM, as the industry boundary of OTI, and performed a preliminary analysis of the structural changes in energy consumption in the Chinese OTI. In studying the energy consumption per unit of industrial added value (EAV) indicator, Xue (2010) believed that IAV was closely linked to product sale and intermediate input prices. Yi (2013) explored the reasons behind the difficulty in analyzing energy consumption and efficiency in the OTI. Yi and explained that the reasons include the non-unified methods in calculating OTI product output and energy consumption and the variations in product categories and classification of subsectors.

The literature survey shows that studies on energy efficiency and analysis of the influencing factors in the textile sector and its subsectors remains limited, especially in China. Therefore, this study seeks to fill these gaps. 


\subsection{Selection of energy efficiency indicators}

Patterson (1996) defines energy efficiency as the ratio of the useful output to the energy input of a progress. A number of energy efficiency indicator systems currently exist worldwide.

The World Energy Council (WEC) has established an energy efficiency indicator system that aims to conduct an international comparative study on energy efficiency and energy conservation policies (WEC, 2001). The indicators of this system can be divided into two types. One type is the economic indicator, and the second is the technical economic indicator or the unit consumption indicator.

The European Union (EU) has also established an energy efficiency indicator system to measure the energy efficiency of member states and their changing trends. The EU system has six macro-indicators (Gvozdenac, 2013). Two of the most important macro-indicators in this system are the ratio between energy and consumption measured in monetary units and energy consumption per production.

Using a systematic introduction, Chinese scholars Wei and Liao (2010) concluded that the energy macro-efficiency indicators that include the energy consumption per unit of GDP, PV, and $\mathrm{AV}$, as well as the physical energy efficiency indicators that include the energy consumption per unit product (EP) and process energy consumption, are the most popularly adopted indicators. The former is commonly used in measuring the total energy efficiency of a country, region, or industry, whereas the latter is more suitable for inter-enterprise with the same structure of production. Wang (2003) performed a comprehensive comparison of the energy efficiency indicators locally and abroad and then highlighted that the energy consumption per unit of GDP was typically used to measure the comprehensive energy efficiency of a country.

The OTI has complex products types, which correspond to various production processes and structures, resulting in inconsistent statistical caliber. Comparing the physical indicators of 
departments with different outputs and calculating the total energy efficiency are challenging tasks. However, the data for economic indicators, such as PV and EAV, are easily accessible and less susceptible to human disturbances. These indicators are suitable in identifying the energy efficiency of some sectors or industries and are widely used in the measurement of single factor energy efficiency. These indicators have gradually become the most commonly used efficiency indicators in Chinese government reports.

In conclusion, economic indicators are chosen to measure the energy efficiency of OTI.

\subsection{Calculation of energy efficiency}

This study selects EPV and EAV as economic indicators to measure the nationwide energy efficiency of OTI in China. EPV refers to the amount of energy consumption per unit of output within a certain period in a country or region and of a sector or an industry. EAV refers to the amount of energy consumption per unit of industrial added value. On the basis of "the general calculation principles of the comprehensive energy consumption" (GB/T 2589-2008), the equation of EPV in this study is as follows:

$$
\mathrm{EPV}=\mathrm{EC} / \mathrm{PV}
$$

where EC is the total energy consumption of the OTI that is converted at the gross coal consumption rate, and PV is the production value within the statistical year.

The following is the equation of EAV:

$$
\mathrm{EAV}=\mathrm{EC} / \mathrm{IAV}
$$

where IAV is the industrial added value, which refers to the final industrial production results of industrial enterprises in monetary terms during the statistical period. Lower EPV and EAV indicate lower energy consumption per unit value within a certain period of time, and consequently, a higher energy macro-efficiency. 


\subsection{Data sources and industry boundary}

Time series variables from 2000 to 2011 are employed in this study. The historical data on energy consumption in the OTI of China are obtained from the China Energy Statistical Yearbooks (DES, 2001-2012), whereas historical data on the gross industrial PV are collected from the China Statistical Yearbooks (NBS, 2001-2012), China Industry Economy Statistical Yearbooks (DIS, 2001-2012), and China Economic Census Yearbooks (SCC, 2004 and 2008). The textile manufacturing data on IAV, production of commodity, and energy consumption in Fujian are all obtained from the Fujian Statistical Yearbooks (FPBS, 2001-2012). The data used in the international comparison are gathered from the US Census Bureau (USCB, 2006; USCB, 2010) and U.S. Energy Information Administration (EIA, 2006; EIA, 2010). The values were translated into the industrial output value at constant prices to ensure comparability of the data. This study converts the PV at the constant price of the textile manufacturing product factory prices using 2000 as the annual base period. For the international comparison, the economic output values are converted to the same monetary unit through the market exchange rate method. In this study, each type of energy is converted into the standard coal equivalent in tons of standard coal equivalent (tce) or million tons of standard coal equivalent (Mtce), based on the conversion coefficient listed in "the general calculation principles of the comprehensive energy consumption" (GB/T 2589-2008).

The OTI is a complicated and heterogeneous industry with multiple subsectors and different enterprises. On the premise of consistent statistical data classification while avoiding the deviation caused by the internal classification adjustment of subsectors, this study identifies TI, CFI, and GSHM as the boundary of the entire textile manufacturing industry, and following the classification of the sectors in the yearbooks.

\section{Energy efficiency in the Chinese textile industry}




\subsection{International comparison}

The US is also a manufacturer that ranks second in the world in terms of manufacturing output (CNTAC, 2013). The manufacturing sectors in the US are divided into three major groups: high-energy, high value-added, and low-energy consumers. OTI belongs to the third group (EIA, 1995). With the US as a representative of the advanced level, this section analyzes the gap between the textile manufacturing in China and that in the US through a horizontal comparison of energy consumption structure and energy efficiency.

Using data on the consumption of all energy types in yearbooks, we analyze the energy structure of the Chinese OTI during the implementation of the "Eleventh Five-year Plan (2006-2010)". The situation in the US is also presented, as shown in Figure 1, in which coal and electric power had a dominant role in the energy composition of OTI in China, accounting for approximately $75 \%$ of the total energy consumption during the past five years. Natural gas accounted for less than $1 \%$ of the total energy consumption of the Chinese OTI. The textile manufacturing in the US depended mainly on gas and electric power, which accounted for more than $80 \%$ of the total energy consumption, and the proportion of coal in the textile energy consumption was very small, at only $13 \%$ in 2006, which decreased further to $7 \%$ in 2010. Moreover, oil, natural gas, and electric power have obvious advantages over coal in terms of efficiency. The calorific value and utilization efficiency of natural gas and oil, which causes less pollution, are both higher than those of coal. In terms of equivalent heat value, the heat of one kilogram coal is also less than that of oil or natural gas, only accounting for $50 \%$ to $60 \%$ of the total heat of oil or natural gas. In particular, the available heat utilization efficiency is only $27 \%$ in processing, conversion, and storage because of low-quality coals and immature technology, whereas of oil and natural gas is $50 \%$ and $57 \%$, respectively, and that of electric energy regarded as clean energy is $85 \%$. With higher quality and less inefficient energy, the energy structure of textile manufacturing in the US is clearly better than that in China. 


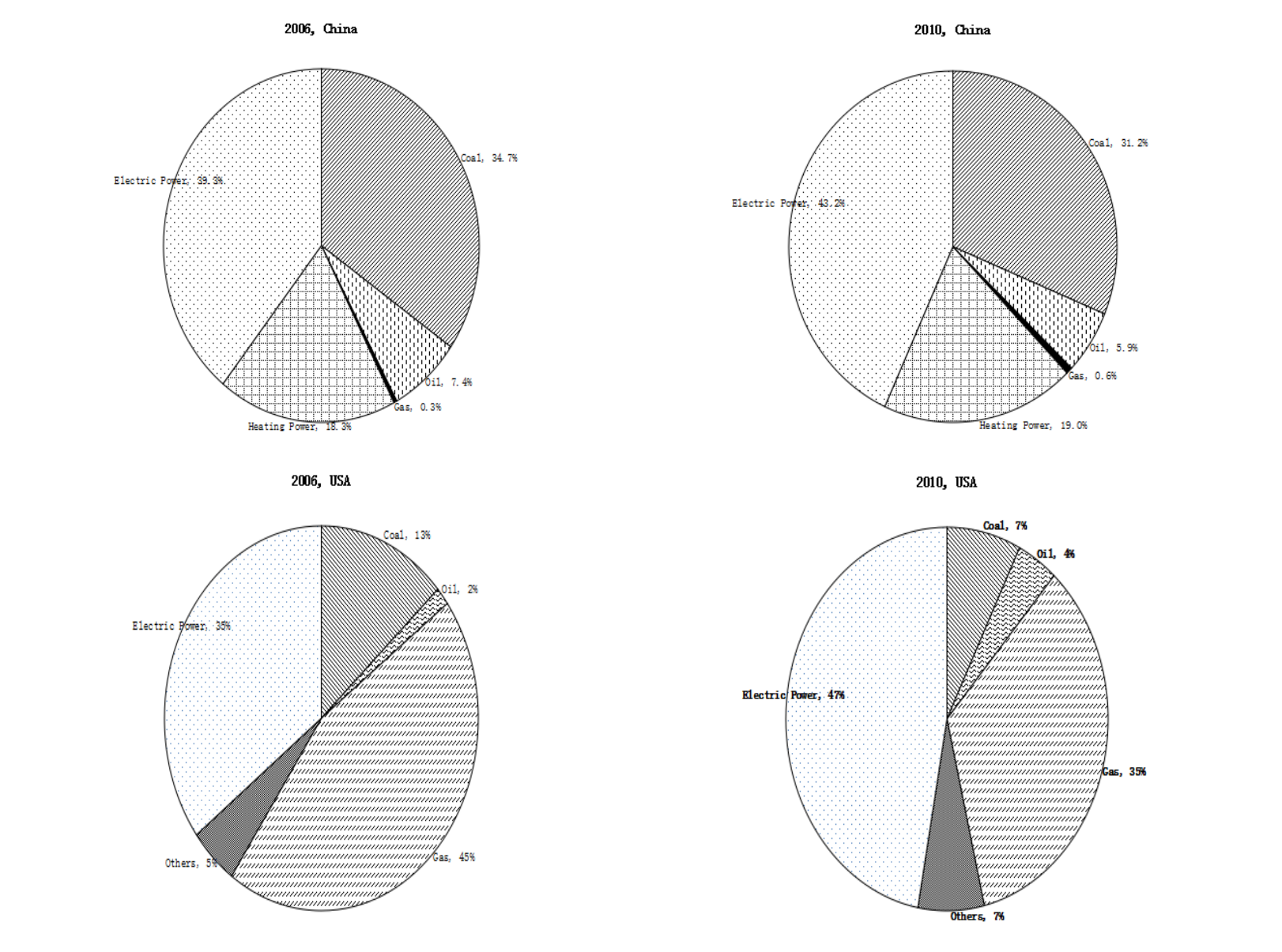

Figure 1. Energy consumption structure of the OTI in China and the US

Figure 2 shows the large energy efficiency gap in OTI between China and the US based on the EAV indicator. The EAV of OTI decreased by nearly $15 \%$ within five years in China, but remained very high compared with that in the US. The EAV of China was 2.13 times than that of the US in 2006 and increased to 2.81 times in 2010. The energy consumption structure had important effects on energy efficiency (Han, 2007). Feng, et al. (2009) indicated that if the proportion of coal in the energy consumption decreased by $1 \%$, energy efficiency would increase by at least $0.303 \%$. The strong link between energy consumption structure and energy efficiency can be obtained by combining the results in Figures 1 and 2. By comparison and to some degree, the lower proportion of coal consumption and higher proportion of gas consumption in the US than that in China contributed to the higher energy efficiency of OTI in the former than in the latter. We also deduce that the economic system may affect the energy efficiency of OTI, which could be one of the 
reasons for the difference between the two countries.

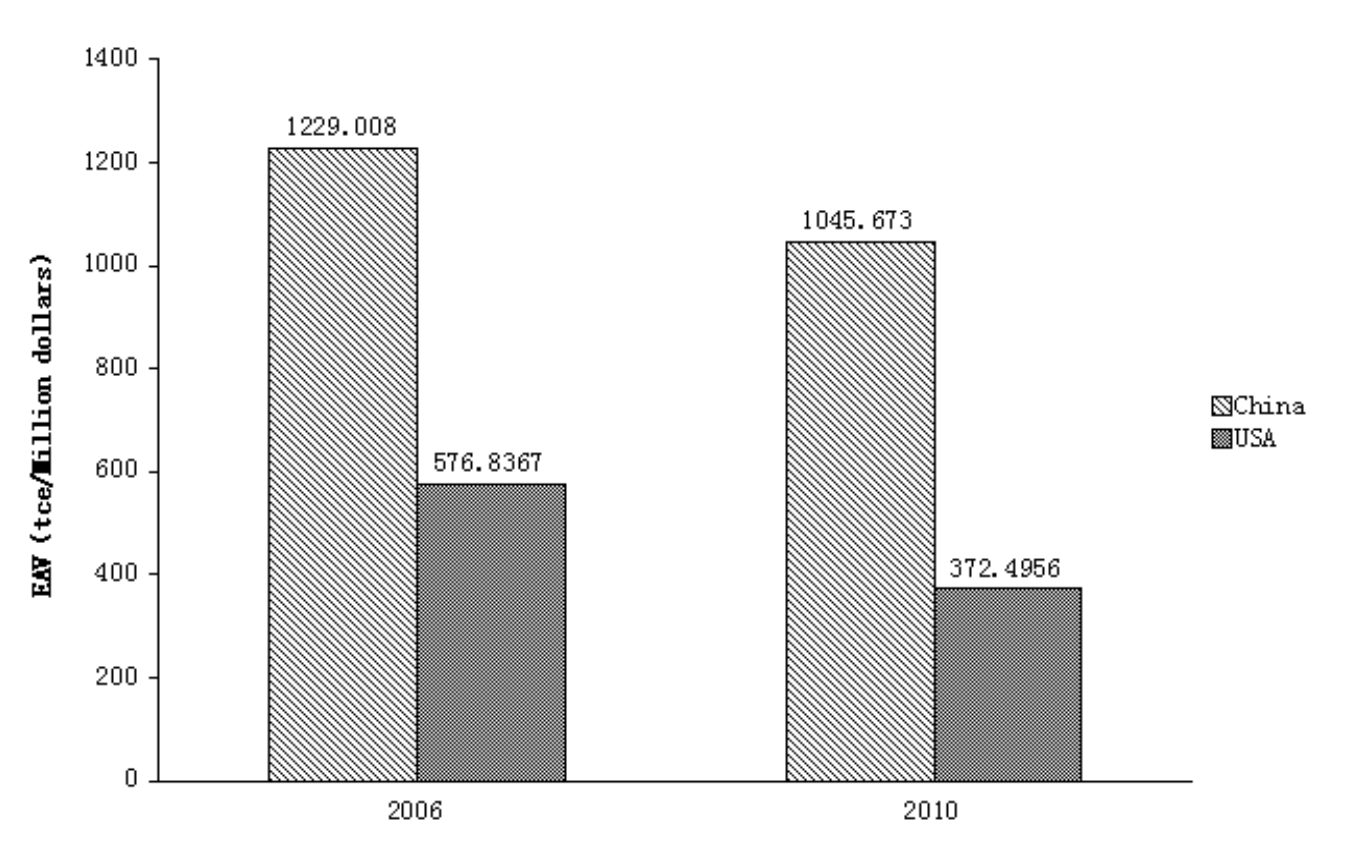

Figure 2. EAV of the OTI in China and the US

\subsection{Energy efficiency comparison among subsectors}

An international comparison shows that the Chinese OTI has experienced industry-wide inefficiency in the last decade and that the government should focus on improving efficiency and productivity. Understanding the energy use of the different industry subsectors is crucial for improving energy-efficiency policies for the OTI. Figure 3 shows the total energy consumption of the OTI from subsector levels in China and the changing trend of this industry over time. Among the subsectors, the energy shares of TI, CFI, and GSHM in the total energy consumption in 2000 were $55.8 \%, 37.5 \%$, and $6.7 \%$, respectively, which changed to $73.3 \%, 17.9 \%$, and $8.8 \%$, respectively, in 2011. From 2000 to 2011, the Chinese OTI experienced a dramatic increase in the rate of energy use and nationwide energy shortages (Zhang et al, 2011; Li et al., 2014). The energy consumption of TI, GSHM, and OTI also increased. The energy consumption in CFI peaked in 2003, but quickly declined in 2004, and thereafter stabilized. In 2004, as product quantity increased, total energy consumption in CFI declined due mainly to the updated technology of CFI, 
ameliorative energy-saving policies, and incentive of higher energy prices. After 2004, the rapid increase in energy prices in China encouraged the promotion of energy conservation and energy efficiency (Jiang, 2009). Energy is one of the main cost factors in the textile industry. In Brazil, energy cost accounted for $5 \%$ of the textile manufacturing cost, whereas in India, energy cost was 12\% (Koç and Kaplan, 2007). Hence, improving energy efficiency should be a primary concern for textile plants, especially during periods of high energy price volatility.

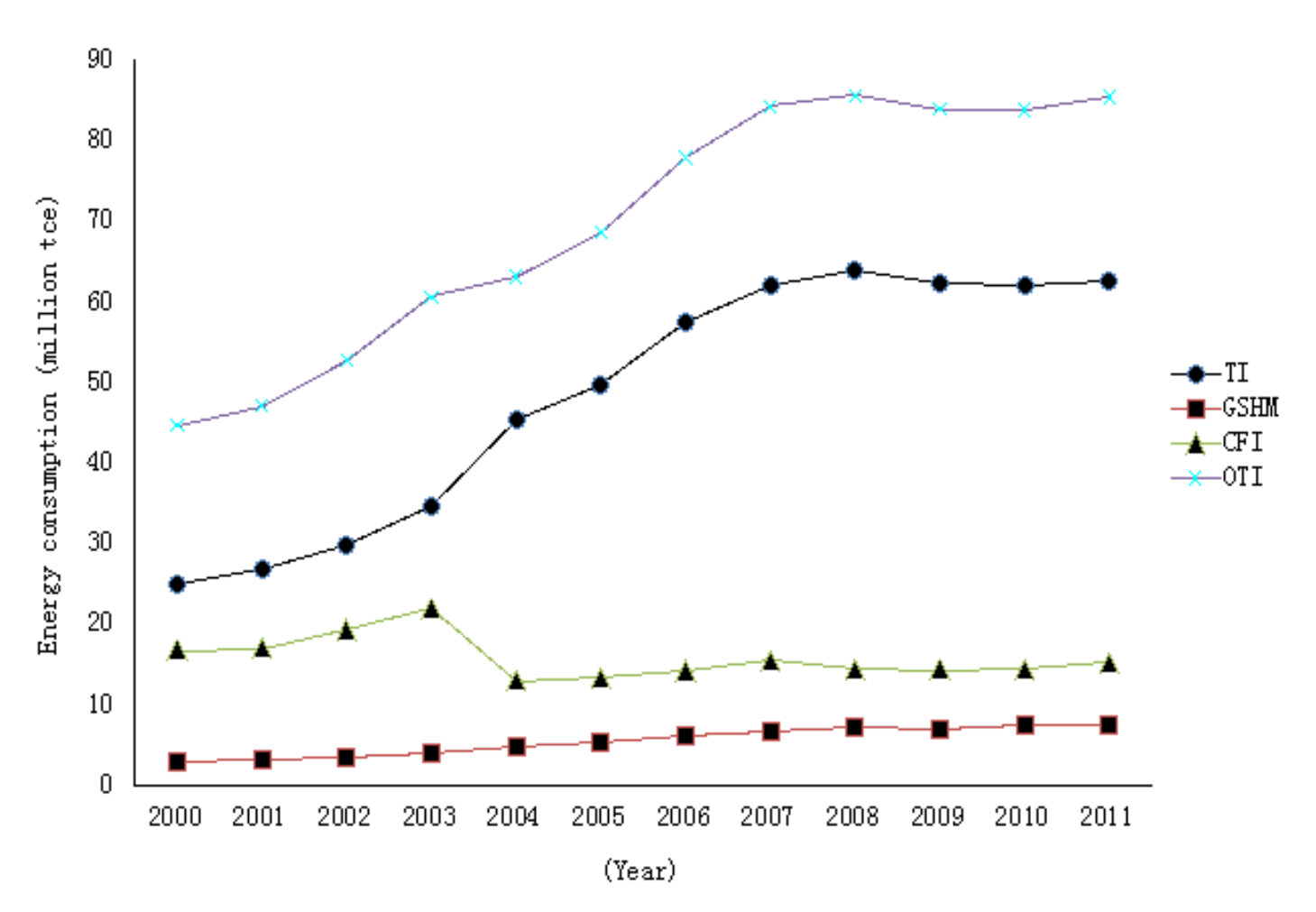

Figure 3. Energy consumption of the OTI in China

Figure 4 shows the energy efficiency levels of three subsectors and the entire OTI based on the EPV indicator. As total energy consumption increased annually, the EPV of OTI declined gradually, and after 2005, the decline rate became slower. The EPV of CFI was the highest for a long time. The average annual EPV of the national CFI was 491.6 tce/million dollars, which was 2.3 times that of the level of the entire OTI and nearly 8 times the level of GSHM, demonstrating that the development of CFI was more dependent on energy than the other two subsectors. The main reason behind this finding could be the low production concentration ratio of $\mathrm{CFI}$ in China, with the 
small-scale enterprises and scattered distribution factories. Previous literature indicated that the chemical fiber production of China accounted for $18.3 \%$ of the world in 1998, whereas number of factories was over $45 \%$ ( $\mathrm{Li}, 2000$ ). In $2012,90 \%$ of the chemical fiber enterprises in China were below the designated size, whereas the other $10 \%$ (approximately 1,870) were above the designated size (Lv, 2012). Moreover, more than $90 \%$ of the enterprises above the designated size were smalland medium-sized enterprises (CNTAC, 2013). The failure to attain the best concentration ratio and manufacturing scale prevented China from promoting energy efficiency (Wang, 2012).

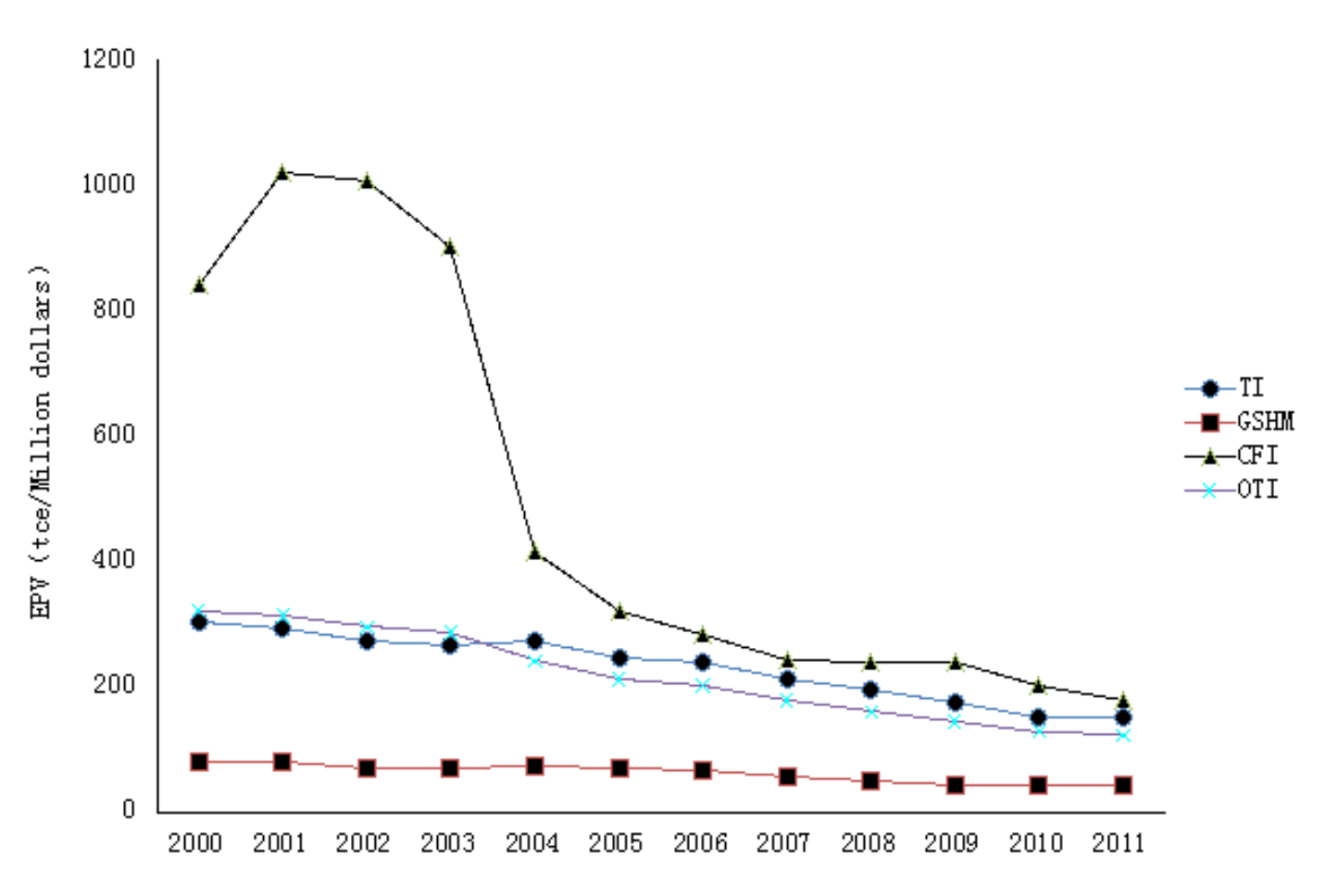

Figure 4. EPV of the OTI in China

In 2004, under increasing PV and yield, the EPV of the national CFI decreased sharply, indicating a remarkable improvement in the energy efficiency of CFI.

As previously mentioned, the change in the energy efficiency of an industry can be attributed to changes in the product structure and efficiency. CFI produces a wide range of cellulosic and synthetic fibers that require different energy levels. Hence, the product structure of CFI should be analyzed to determine the dominant factors that cause a sharp decrease in the indicators. The 
product structure of CFI in 2004 exhibited a proportion of main products based on the China Textile Industry Development Report (CNTAC, 2002-2006). Standard and relative standard deviations are adopted to determine the stability of the product structure (Table 1). Synthetic fibers accounted for $93 \%$ of CFI, and its standard deviation was as low as $0.14 \%$. Polyester fiber emerged as the most widely used synthetic fiber, accounting for $80.97 \%$ of CFI, with a small standard deviation and relative standard deviation. Table 1 shows that the CFI product structure around 2004 was stable. The sharp decrease in indicators was caused mainly by efficiency change. Further analysis of the efficiency change drivers showed that improvements in energy efficiency in the Chinese CFI are driven potentially by the overall industry diffusion of advanced technology. According to the analysis in the China Industry Annual Report for Chemical Fiber Sectors in 2004 (CEIN, 2004), considerable changes occurred in CFI as science and technology developed rapidly in the 21st century. Private enterprises have recently joined the industry, resulting in a more thorough development of potentialities and synergies and the promotion of technical progress.

Table 1. CFI product mix from 2002 to 2006.

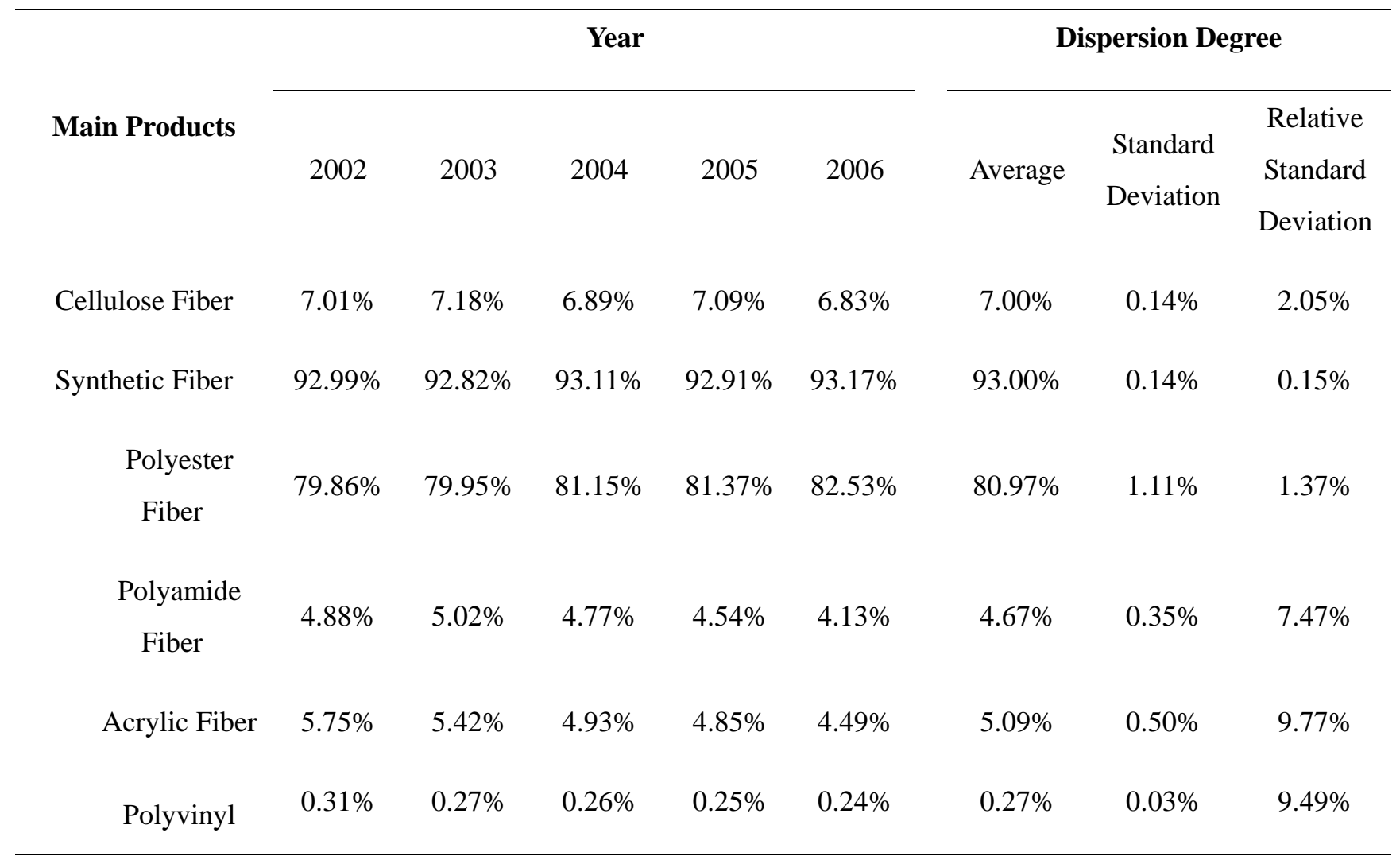


Fiber

Polypropylene

Fiber

$2.20 \%$

$2.16 \%$

$2.00 \%$

$1.89 \%$

$1.79 \%$

$2.01 \%$

$0.17 \%$

$8.71 \%$

During the implementation of the "Eleventh Five-year Plan," the proportion of accounted chemical fibers in textile materials was $70 \%$, and chemical fiber was one of the nine major energy-intensive export products in China (Jiang, 2009). The top five energy-intensive petrochemical industries in China belong in CFI. As analyzed previously, energy conservation and emission reduction efforts of CFI are imminent and urgent, and thus, energy efficiency of CFI must be highlighted.

\subsection{Energy efficiency of CFI in Fujian Province}

This section measures the energy efficiency of the key subsector in the key area, namely, Fujian Province (Figure 5) and compares the level of energy efficiency of the Fujian Province with that of the national efficiency level. Figure 5 shows that the IAV of CFI in Fujian generally increased, whereas EAV declined. The IAV of CFI in Fujian increased sevenfold from 2000 to 2011. The EAV of CFI reduced by $78 \%$ during the research period. Figure 5 illustrates that since 2004, the IAV of CFI in Fujian decreased significantly simultaneous with the national level, which was an advantage in the technology improvement of this sector. 


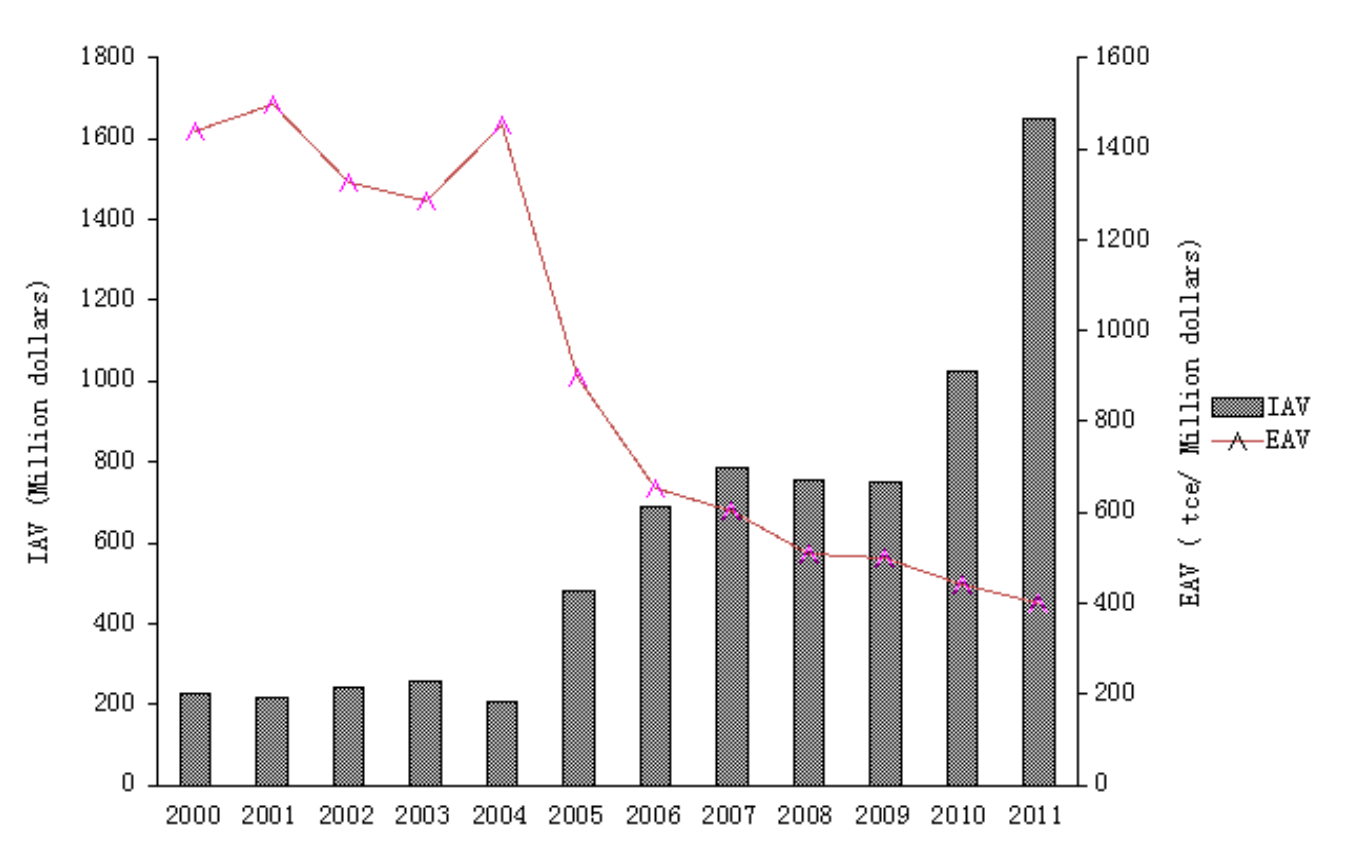

Figure 5. IAV and EAV of the CFI in Fujian

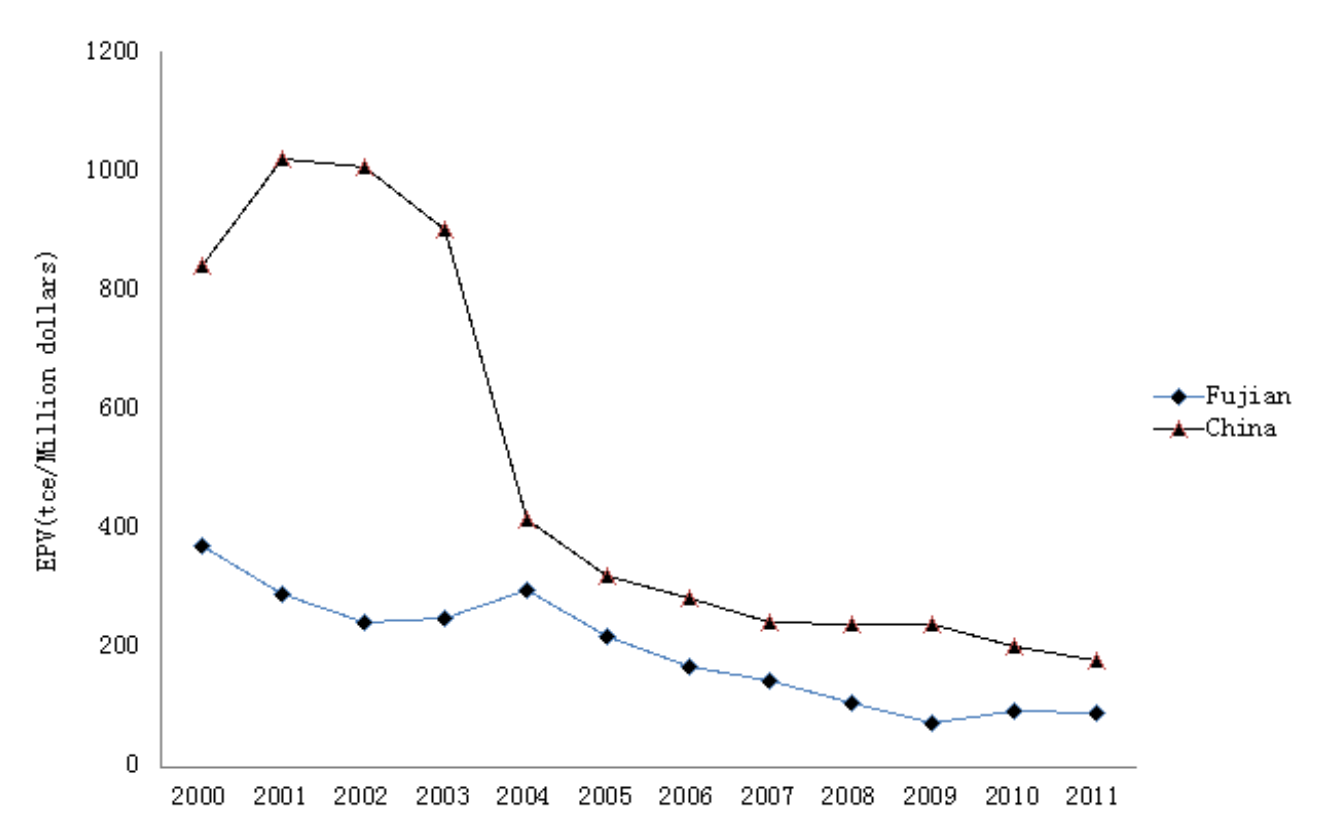

Figure 6. EPV of CFI in Fujian and China

Figure 6 shows that the EPV of CFI in Fujian was below the national average. The average annual EPV level of Fujian was only $39.7 \%$ of the national average level, thereby suggesting that 
the aim to promote energy efficiency in Fujian ranked first in China. Hence, factors affecting the energy efficiency of CFI in the national level and Fujian provincial region should be explored.

\section{Influencing factors analysis}

\subsection{Analysis of factors influencing energy efficiency of CFI in China}

We can qualitatively deduce from the comparison described in Section 3 that the factors influencing energy efficiency of CFI include economic system variable (ESV), energy structure (ES), industry concentration ratio (ICR), industry scale of a sector (IS), and technical level (TL), among others. This section will establish the econometric model to analyze quantitatively and test the influence of these factors on the energy efficiency of Chinese CFI. The model variables are explained and expressed as follows.

ESV refers to the economic system variable, which is the proportion of the output value of state-owned and state-holding enterprises to the total industrial output CFI. ES is the proportion of coal consumption in the total energy consumption of CFI, which determines the energy consumption structure. ICR represents the industry concentration ratio, which is the proportion of large- and medium-sized enterprise output value in the total industrial output CFI. IS represents the industry scale and is the result of dividing the total industrial output value by the number of enterprises of CFI. The unit of IS is one hundred million Yuan/individual. TL is the annual logarithm value of the number of patent applications in CFI.

Table 2 The descriptive statistical characteristics of factors influencing energy efficiency

\begin{tabular}{cccccc}
\hline Variable & ESV & ES & ICR & IS & TL \\
\hline Minimum & 0.0820 & 0.3420 & 0.7140 & 1.1710 & 2.0680 \\
Maximum & 0.5500 & 0.5980 & 0.7930 & 3.0320 & 3.1100 \\
Median & 0.2138 & 0.5017 & 0.7560 & 1.7956 & 2.4313 \\
\hline
\end{tabular}




\begin{tabular}{|c|c|c|c|c|c|}
\hline Mean & 0.2372 & 0.4809 & 0.7590 & 1.7902 & 2.5006 \\
\hline Standard deviation & 0.1447 & 0.0795 & 0.0245 & 0.5290 & 0.3443 \\
\hline
\end{tabular}

Note: Data were extracted from the "China Statistical Yearbook (2001 to 2012)."

Using EPV as the dependent variable index, we can obtain the CFI energy efficiency influencing factors model in China as follows:

$$
\mathrm{Y}=\mathrm{C}+\alpha_{1} \mathrm{ESV}+\alpha_{2} \mathrm{ES}_{2}+\alpha_{3} \mathrm{ICR}+\alpha_{4} \mathrm{IS}+\alpha_{5} \mathrm{TL}+\mu
$$

In the equation, to reduce heteroscedasticity and colinearity between variables, $\mathrm{Y}$ is the logarithm value of EPV, $\mathrm{C}$ is a constant term, $\alpha$ is parameter coefficient, and $\mu$ is the residual term.

Backward elimination method is part of the stepwise regression analysis, which gradually excludes the most insignificant argument from the total arguments until all independent variables became significant. Thus, the final result is the "optimal" equation. The study used SPSS 20.0 software to create a multiple regression equation by backward regression. The results can be found in Table 3.

Table 3. Regression results of key influencing factors of CFI energy efficiency in China

\begin{tabular}{ccccc}
\hline Variable & Coefficient & T-Statistic & Prob. & VIF \\
\hline C & $4.497 * *$ & 12.628 & 0.000 & \\
ESV & $0.554^{*}$ & 2.517 & 0.040 & 3.975 \\
ES & $1.576^{* *}$ & 6.130 & 0.000 & 2.820 \\
IS & $-0.139 *$ & -2.753 & 0.028 & 1.640 \\
TL & $-0.400^{* *}$ & -3.876 & 0.006 & 4.960 \\
\hline
\end{tabular}

Note: $* *$ indicates significant at $1 \%$ level. * indicates significant at 5\% level.

The regression shows that the R-squared for testing the goodness of fit of the regression equation is 0.978 , and the adjusted R-squared is 0.966 . Both results are very close to 1 . Hence, the 
goodness of fit is high. The value of Durbin-Watson (DW) is 2.237, indicating no correlation between the residuals. The obtained equation is in line with the colinearity test.

From Table 3, we can deduce that the explanatory variable ICR is not covered by the model, which implies that industrial concentration does not have a significant influence on energy efficiency, as speculated. The explanatory variables, ES and TL, passed the significance tests at $1 \%$ level, whereas ESV and IS passed the significance tests at 5\% level. Low EPV indicates high energy efficiency. These findings suggest the following conclusions.

In the market, the proportion of output value of state-owned and state holding enterprises (ESV) increased by $1 \%$ and EPV (in logs) of CFI increased by $0.554 \%$, indicating that the high proportion of state-owned economy is not conducive to improving the energy efficiency of CFI. Hence, better reforms in state-owned enterprises should be implemented and the development of non-public enterprises, such as private and foreign-funded enterprises, should be encouraged. Moreover, the "State Retreats While Private Sector Advances" stock reform should be promoted to strengthen the enterprise incentive mechanism from the micro perspective by spontaneously implementing the economic behavior of improving manufacturing and operations efficiency and saving scarce resources and energy. The proportion of coal (ES) in the energy consumption structure increase will improve the EPV (in logs) of the Chinese CFI, with a high regression coefficient of 1.576. China should actively explore measures that will improve the efficiency of its coal utilization and promote highly efficient and environment-friendly and renewable energy. Hence, structures should gradually strike a path toward the optimization direction based on high-quality, low-carbon, and clean energy, which is important for the sustainable development of Chinese CFI.

A negative correlation exists between the explanatory variables IS, TL, and the dependent variable index, which indicates that increasing the industry scale and technical level will decrease the EPV of CFI. Among them, IS is an important factor that affects CFI energy efficiency. The expansion of enterprise scale and increase in energy efficiency can help bring out the potentials of 
equipment and intensification of energy. Currently, many small- and medium-sized chemical fiber enterprises and small workshops have a significant influence in improving the energy efficiency of CFI. The improvement of TL will also decrease the EPV of CFI, which has greater influence than IS. Technological advances enhance the efficiency of the production process recognized by the academia. The results of the CFI energy efficiency factor analysis are aligned with objective laws. Currently, the production process and technology of CFI in China are relatively backward. Thus, the government must establish industrial policies and related regulations to guide enterprises in investing more in environmental protection technology, introducing technical knowledge and management experience, and in creating new invention technologies to promote technological advances that could improve the energy efficiency of CFI.

\subsection{Comparison of factors influencing energy efficiency of Fujian's CFI}

After identifying the main factors influencing energy efficiency in China's CFI, we compare the factors identified at the national level with those in Fujian, a province in China. This comparison will explain the differences on energy efficiency between Fujian and China.

Table 4 shows that ESV of CFI in Fujian is only 0.0901, which is $37.9 \%$ of that in China. In other words, the non-public enterprises of Fujian's CFI has more development advantages because Fujian's location in a coastal region and as the western principal part of the Straits Economic Zone. This location has enabled the province to attract considerable foreign capital. Fujian also has Xiamen, a special economic zone that benefits from free trade. The coal consumption share of CFI in Fujian is 0.11 less than the national average, indicating the higher ES of CFI in Fujian. Moreover, the enterprise scale of Fujian is also larger than that of the national level. On average, the annual output value of each enterprise in Fujian is about 10 million Yuan more than that of the national level. Small and medium enterprises are dominant business forms of the CFI in China. In recent years, Fujian exerted considerable efforts to increase the capacity concentration in the province. Fujian has gradually formed TI clusters in accordance with the regional economic features of local 
cities, counties, or towns and a number of powerful enterprises in the province have also merged to become larger and more competitive chemical fiber clients. The industry agglomeration in Fujian's CFI promotes more efficient energy use in the production of the same products. With the lack of historical data in this industry, the number of patents generated in CFI each year in Fujian cannot be determined. However, the growth rate on the number of patents in this industry is 1.2 times greater than that of the national average. Hence, the technological development in Fujian could be considered as being at the forefront in China. In addition, under the circumstances of technological advancement of the nationwide OTI, the Fujian textile manufacturing enterprises took a further step and introduced a larger number of latest technologies and equipment available locally and abroad. The industrial innovation capability in the province is also constantly improving, and the capability of the enterprises for research and development is being strengthened continuously. A group of technology development centers and a manufacturing base have been established in the province. The technological progress in Fujian provides strong support for the energy conservation and energy efficiency efforts of Fujian's CFI.

Table 4. Comparison of factors influencing EPV of CFI between Fujian and China

\begin{tabular}{lcccc}
\hline & ESV & ES & IS & TL \\
\hline Function direction & + & + & - & - \\
Average value in China & 0.2372 & 0.4809 & 1.7902 & 2.5006 \\
Average value in Fujian & 0.0901 & 0.3783 & 1.8846 & $/$ \\
\hline
\end{tabular}

\section{Conclusions and policy implications}

Energy efficiency is closely connected with the sustainable development of an industry. This study assesses the energy efficiency of OTI in China. Compared with OTI energy efficiency in other countries, the energy efficiency in China's OTI remains far from the advanced level because of the disadvantages of the energy consumption structure, which consists of a large percentage of coal and 
a small proportion of other types of excellent quality and efficient energy. However, through years of effort, the energy efficiency of China's OTI has increased significantly. A transverse comparison analysis of energy efficiency in the major subsectors of OTI in China indicates that GSHM has the highest energy efficiency, whereas that of CFI is the lowest. CFI is analyzed carefully as the key subsector of OTI. The results of the quantitative regression analysis indicate that the main factors influencing the energy efficiency of CFI are ESV, ES, IS, and TL, whereas ICR did not play an obvious role. Effective technological advancement is also found to contribute significantly in increasing the energy efficiency in CFI, whereas the proportion of coal consumption in the energy consumption had a considerable role in reducing the energy efficiency of CFI. The energy efficiency and the factors that influence efficiency in the local and national major subsectors, such as CFI, are compared separately. Both EAV and EPV indicators show that the energy efficiency of Fujian's CFI continues to improve each year and is currently above the national average. The lower proportion of output value of state-owned and state proprietary enterprises, lower share of coal in energy consumption structure, industrial scale with a majority of medium- and large-sized enterprises, and higher TL are the main reasons for the successful energy efficiency of the Fujian's CFI.

Industry is the starting point of the national energy policy. Energy efficiency provides essential information that supports the creation of scientifically sound energy policies for the various industries. Based on an analysis of energy efficiency, this paper argues that policy changes are necessary to achieve further development of energy efficiency in OTI in China, particularly in CFI. Measures such as economic structure reform, ES, IS, and energy-saving technology should be undertaken. The industrial structure requires optimization, which can be achieved by reducing the proportion of CFI and increasing the share of GSHM. The economic structure should also be optimized further by reducing the proportion of state-owned enterprises and increasing non-public enterprises. The energy consumption structure also requires optimization, which can be achieved by 
reducing the proportion of coal and oil, and using clean and renewable energy as a substitute. CFI can be assisted in realizing its merit scale by encouraging small- and medium-sized enterprises to cluster and consolidate to form large and medium-sized enterprises, thereby improving the efficiency of energy use. The development and application of energy-saving technologies of subsectors should be emphasized to achieve the aims of technical consumption reduction and improvement of the energy efficiency of the entire industry. The management experience, technology, and advantages of Fujian's CFI should be popularized to the entire country. The energy policy should also be strengthened further, and should be one of the top priorities of both national and local governments.

In conclusion, improving the energy efficiency of China's OTI will provide better opportunities for energy conservation and build a more scientific energy management system.

\section{Acknowledgments}

The authors would like to thank National Bureau of Statistics (NBS), Fujian Provincial Bureau of Statistics (FPBS), the Department of Energy Statistics (DES), the Department of Industry Statistics (DIS), the China National Textile and Apparel Council (CNTAC) for their data accessibility for this study.

\section{References:}

Blesl, M., Das, A., Fahl, U., Remme, U., 2007. Role of energy efficiency standards in reducing $\mathrm{CO}_{2}$ emissions in Germany: an assessment with TIMES. Energy Policy 35, 772-785.

China Economic Information Network (CEIN), 2004. China Industry Annual Report for Chemical Fiber Sectors in 2004. Available at:

http://cei.56.org.cn/doc/BGSASAC/pdf/2004/200410.pdf $\quad$ (accessed July 19, 2014). (in Chinese) 
Cagno, E., Trianni, A., 2012. Analysis of the most effective energy efficiency opportunities in manufacturing primary metals, plastics, and textiles small- and medium-sized enterprises. J. Energy Resour. Technol 2012, 134: 1-9.

Chen, G., 2013. Variation coefficient analysis of energy consumption structure of China's textile industry. China Textile, 8, 134-135. (in Chinese)

Cui, Q., Kuang, H., Wu, C., Li, Y., 2014. The changing trend and influencing factors of energy efficiency: The case of nine countries. Energy 64, 1026-1034.

China National Textile and Apparel Council (CNTAC), 2002-2013. China textile industry development report. China Textile Press, Beijing. (in Chinese)

Department of Energy Statistics (DES), 2001-2012. China Energy Statistical Yearbook. China Statistic Publishing House, Beijing. (in Chinese)

Department of Industry Statistics (DIS), 2001-2012. China Industry Economy Statistical Yearbook. China Statistic Publishing House, Beijing. (in Chinese)

Energy Information Administration (EIA), 1995. Measuring Energy Efficiency in the United States' Economy. Available at: http://www.eia.gov/emeu/efficiency/ee_report_html.htm (accessed July 19, 2014).

Energy Information Administration (EIA), 2006. Manufacturing Energy Consumption Survey Data. Available at: http://www.eia.gov/consumption/manufacturing/data/2006/pdf/Table1_2.pdf (accessed July 19, 2014).

Energy Information Administration (EIA), 2010. Manufacturing Energy Consumption Survey Data. Available at: http://www.eia.gov/consumption/manufacturing/data/2010/pdf/Table1_2.pdf (accessed July 19, 2014).

Fujian Provincial Bureau of Statistics (FPBS), 2001-2012. Fujian Statistical Yearbook. China 
Statistic Publishing House, Beijing. (in Chinese)

Feng, T.W., Sun, L.Y., Zhang, Y., 2009. The relationship between energy consumption structure, economic structure and energy intensity in China. Energy Policy, 37, 5475-5483.

Gvozdenac, D., 2013. Energy Efficiency Indicators. 2013. Available at: http://www.peec.uns.ac.rs/iNTeg\%20Risk/Energy\%20Efficiency\%20Indicators.pdf. (accessed July 19, 2014).

Hasanbeigi, A., 2010. Energy-efficiency improvement opportunities for the textile industry. Berkeley, CA: Lawrence Berkeley National Laboratory, 2010. Available at: http://escholarship.org/uc/item/6jw8s2gz (accessed July 19, 2014).

Hasanbeigi, A., Price, L., 2012a. A review of energy use and energy efficiency technologies for the textile industry. Renew. Sust. Energ. Rev., 16, 3648-3665.

Hasanbeigi, A., Hasanabadi, A., Abdorrazaghi, M., 2012b. Comparison analysis of energy intensity for five major subsectors of the textile industry in Iran. J. Clean. Prod. 23, 186-194.

Hong, G.B., Su, T.L., Lee, J.D., et al., 2010. Energy conservation potential in Taiwanese textile industry. Energy Policy 38, 7048-7053.

Han, Z.Y., Fan, Y., Jiao, J.L., et al., 2007. Energy structure, marginal efficiency and substitution rate: An empirical study of China. Energy, 32, 935-942.

International Energy Agency (IEA), 2007. Tracking industrial energy efficiency and $\mathrm{CO}_{2}$ emissions. Available at: http://www.iea.org/Textbase/npsum/tracking2007SUM.pdf. (accessed July 19, 2014).

International Energy Agency (IEA), 2012. Key World Energy Statistics. Available at: http://www.iea.org/publications/freepublications/publication/kwes.pdf. (accessed July 19, 2014). 
International Energy Workshop (IEW), 2013. Bottom-up Assessment of Chinese Manufacturing Growth and Energy Use up to 2020. Available at: http://www.internationalenergyworkshop.org/docs/IEW\%202013_3A1paperHasanbeigi.pdf (accessed July 19, 2014).

Intergovernmental Panel On Climate Change (IPCC), 2007. IPCC Fourth Assessment Report: Climate Change 2007. Available at: http://www.ipcc.ch/publications_and_data/publications_and_data_reports.shtml\#1 (accessed July 19, 2014).

Jiang, K.J., 2009. Energy efficiency improvement in China: a significant progress for the 11th Five Year Plan. Energ Effic 2, 401-409.

Koç, E., Kaplan, E., 2007. An investigation on energy consumption in yarn production with special reference to ring spinning. Fibers Text East Eur 15, 18-24.

Lenzing, 2013. Annual Report 2013. Available at: http://www.lenzing.com/fileadmin/template/pdf/konzern/geschaftsberichte_gb_ugb_jfb/GB_EN/GB 2013_EN.pdf (accessed July 19, 2014).

Li, R., 2000. Current situation of the chemical fiber industry in China and suggest ions for its development. Modern Chemical Industry, 03, 1-4. (in Chinese)

Lin, R.Q., 2002. The policy and strategic choice of Fujian in the face of green textiles. The Light \& Textile Indu, 06, 11-14. (in Chinese)

Lv, Z., 2012. Industry Yearbook. China Financial and Economic Publishing House, Beijing. (in Chinese)

Martínez, C.I.P., 2009. Energy efficiency developments in the manufacturing industries of Germany and Colombia, 1998-2005. Energy Sustaina Dev 13, 189-201. 
Mohsen, M.S., Akash, B.A., 1996. Energy analysis of the steel making industry. Int. J. Energ. Res. 22, 1049-1054.

National Bureau of Statistics (NBS), 2001-2012. China Statistic Yearbook. China Statistic Publishing House, Beijing. (in Chinese)

Ozturk, H.K., 2005. Energy usage and cost in textile industry: A case study for Turkey. Energy 30, 2424-2446.

Palanichamy, C., Babu, N.S., 2005. Second stage energy conservation experience with a textile industry. Energy Policy, 33, 603-609.

Patterson, M.G., 1996. What is energy efficiency? : Concepts, indicators and methodological issues?. Energy Policy 24, 377-390.

Palamutcu, S., 2010. Electric energy consumption in the cotton textile processing stages. Energy, 35, 2945-2952.

Reddy, B.S., Ray, B.K., 2011. Understanding industrial energy use: physical energy intensity changes in Indian manufacturing sector. Energy Policy, 39, 7234-7243.

State Council of China (SCC), 2004, 2008. China Economic Census Yearbooks. China Statistic Publishing House, Beijing. (in Chinese)

Sue Wing, I., 2008. Eckaus R S. Explaining Long-Run changes in the energy intensity of the US economy. Available at: http://dspace.mit.edu/bitstream/handle/1721.1/5545/MITJPSPGC_Rpt116.pdf?sequence=1 (accessed July 19, 2014)

Trianni, A., Cagno, E., Thollander, P., Backlund, S., 2013. Barriers to industrial energy efficiency in foundries: a European comparison. J. Clean. Prod. 40, 161-176. 
Tanaka, K., 2008. Assessment of energy efficiency performance measures in industry and their application for policy. Energy Policy 36, 2887-2902.

United States Census Bureau (USCB), 2006. 2006 Statistics for Industry Groups and Industries. Available at: http://factfinder2.census.gov/faces/tableservices/jsf/pages/productview.xhtml?pid=ASM_2006_31G $\underline{\text { S101\&prodType=table }} \quad$ (accessed July 19, 2014).

United States Census Bureau (USCB), 2010. 2010 Statistics for Industry Groups and Industries. Available at: http://factfinder2.census.gov/faces/tableservices/jsf/pages/productview.xhtml?pid=ASM_2010_31G $\underline{\text { S101\&prodType=table }}$ (accessed July 19, 2014).

Utlu, Z., Hepbasli, A., 2007. A review on analyzing and evaluating the energy utilization efficiency of countries. Renew. Sust. Energ. Rev., 11, 1-29.

Wei, C., 2010. Industrial energy efficiency, energy saving potential and influencing factors- the empirical analysis based on Zhejiang province. Study and Practice, 3, 16-25. (in Chinese)

World Energy Council (WEC). Energy Efficiency Polices and Indicators. 2001.

Wang, K., Wei, Y.M., Zhang, X., 2013. Energy and emissions efficiency patterns of Chinese regions: A multi-directional efficiency analysis. Appl. Energ., 104, 105-116.

Wang, Q.Y., 2003. China's energy efficiency and international comparison. Energy Conservation and Environmental Protection. 8, 5-14. (in Chinese)

Wu, A.H., Cao, Y.Y., Liu, B., 2014. Energy efficiency evaluation for regions in China: an application of DEA and Malmquist indices. Energ. Effic. 7, 429-439.

Zhang, H.W., Lan, Z.R., 2013. Study on the Evaluation Index System of Regional Energy Use Efficiency. Adv. Mater. Res. 734, 1757-1765. 
Zhang, N., Lior, N., Jin, H., 2011. The energy situation and its sustainable development strategy in China. Energy, 36, 3639-3649.

Zhao, X.L., Ma, Q., Yang, R., 2013. Factors influencing $\mathrm{CO}_{2}$ emissions in China's power industry: Co-integration analysis. Energy Policy. 57, 89-98. 